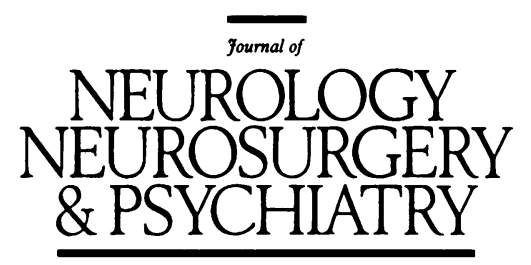

Editorial

\title{
Multiple sclerosis and vitamin B12 metabolism
}

Our understanding of the neurology of vitamin B12 deficiency has evolved slowly over more than a century. ${ }^{1-3}$ Multiple sclerosis (MS) is usually clinically and pathologically clearly distinguishable from vitamin B12 deficiency, although demyelination is a prominent feature of both conditions. Recent reports ${ }^{45}$ of the occasional association of multiple sclerosis and vitamin B12 deficiency have revived interest in vitamin B12 metabolism in MS and raised questions about the nature of the association.

Vitamin B12 deficiency is usually a disorder of middle or late life and therefore rarely occurs in the typical age range of presentation of MS. $^{6}$ Furthermore the vitamin B12 deficiency associated with MS had other unusual features. Although pernicious anaemia (PA) is the commonest cause of vitamin B12 deficiency, this was present in only two, possibly three, of the ten cases described by Reynolds et al..$^{5}$ In most cases the cause of the deficiency was uncertain and a disorder of vitamin B12 binding or transport was suspected. The diagnosis both of the deficiency state and of the MS were not in doubt and none of the cases had the typical neurological manifestations of vitamin B12 deficiency.

Subsequently Crellin et $a l^{7}$ reviewed the records of patients with definite MS admitted to hospital in one year and found they had mild but significant macrocytosis compared with an age and sex matched neurological control group admitted to the same ward. This was confirmed by Najim Al-Din et $a l^{8}$ who also noted that in most patients the macrocytosis was present near the time of presentation of the MS and was therefore unlikely to be simply the consequence of chronic disease. Both investigations support old but long-forgotten studies showing a slight increase in red cell size in MS. ${ }^{910}$ In a prospective study of patients with MS Reynolds et al ${ }^{11}$ found significant lowering of serum vitamin B12 and elevation of plasma unsaturated R-binder capacity, the latter occasionally to very high values. The function of R-binder is unknown, but it is interesting that there are three case reports in the literature of a possible association of MS and R-binder deficiency. ${ }^{12}{ }^{13}$ There is very little vitamin B12 detectable in normal CSF and standard assay procedures are not sensitive enough to detect changes in CSF vitamin B12 in disease. Employing a new more sensitive technique Njist $e t$ al $^{14}$ reported a significant lowering of CSF vitamin B12 in MS. Although the fall in serum vitamin B12 in these patients was not significant there was a significant correlation between serum and CSF vitamin levels. Some individuals may, however, have a low CSF vitamin B12 with a normal serum vitamin B12. Until recently the diagnosis of vitamin B12 deficiency has been very dependent on the serum vitamin B12 assay, but there is evidence that this is not an entirely reliable guide to tissue vitamin B12 deficiency. ${ }^{15}$ New evidence suggests that total plasma homocysteine may be a useful guide to functional vitamin B12 deficiency. ${ }^{15}$ In our patients with MS, plasma homocysteine levels were significantly elevated and negatively correlated with serum vitamin B12, suggesting that low levels of serum vitamin B12 in MS are associated with functional deficiency even in the absence of overt haematological manifestations of deficiency. ${ }^{16}$

Although the majority of patients with MS do not have detectable vitamin B12 deficiency there is evidence of an overlap of the two disorders and a subgroup of patients with this association. It is very unlikely that the association is coincidental. On the basis of epidemiological and age considerations the risk of the two disorders occurring together by chance is remote. ${ }^{5}$ One possibility is that the combined occurrence of MS and vitamin B12 deficiency represents an increased association of two autoimmune disorders. It is remarkable that, despite the fact that the age range is so different, the sex, racial and geographical distribution of MS and PA are similar. ${ }^{57}$ Thus PA has a higher prevalence in northern Europe compared with southern Europe and tropical areas, a diminishing prevalence from the north to the south of the UK and a higher incidence in white than black North Americans. ${ }^{6}$ The female-to-male ratio in both disorders is 1.3:1. Najim AlDin $e t l^{8}$ have also drawn attention to some similarities in the HLA associations of MS and PA. Two other possible explanations for the association between MS and vitamin B12 deficiency are: 1) That vitamin B12 deficiency, from whatever cause, renders some patients more vulnerable to the putative viral/immunological mechanisms widely suspected in $\mathrm{MS}^{5}$ or 2) That chronic immune reactions or recurrent myelin repair processes increase the demand for vitamin B12. ${ }^{11}$ The latter possibility might explain the occurrence of low CSF vitamin B12 levels in the presence of normal serum values. Furthermore, impressive evidence of a probable role for vitamin B12 in myelin formation comes from recent studies of children with inborn errors of vitamin B12 and methylation metabolism. ${ }^{17}$ An important aspect of the neuropathology of these children is a failure of myelination, or even demyelination. Appropriate treatment can result in MRI evidence of myelination or remyelination.

On first reflection it may seem rather unlikely that a deficiency that usually leads to various well known 
progressive neurological syndromes ${ }^{3}$ should also cause a previously unrecognised relapsing and remitting syndrome, all the more so as there is thought to be a clear distinction between the "inflammatory" neuropathology of MS and the non-inflammatory neuropathology of vitamin B12 deficiency. Before rejecting a causal hypothesis in this subgroup a few puzzling observations should be borne in mind: 1) In clinical practice the distinction between MS and "subacute combined degeneration" (SCD) due to vitamin $\mathrm{B} 12$ deficiency occasionally leads to difficulty and uncertainty; 2) There are some interesting reports in the literature of patients in whom a diagnosis of MS has been made or suspected only for the authors to change the diagnosis to SCD following the discovery of an abnormality of vitamin Bl2 metabolism; $;^{1318}$ 3) None of the patients with MS and unequivocal vitamin $\mathrm{Bl} 2$ deficiency have the expected typical neurological manifestations of this deficiency state. ${ }^{5}$ In particular, none of them had clinical or electromyographic evidence of peripheral neuropathy, the commonest neurological manifestation of vitamin $\mathrm{Bl} 2$ deficiency; ${ }^{3}$ 4) Demyelination is a prominent feature of the neuropathology of vitamin B12 deficiency in the spinal cord (SCD) and brain, and of inborn errors of vitamin B12 metabolism; ${ }^{17}$ 5) Recently a myelopathy which neuropathologically resembles SCD has been described in AIDS patients in the absence of vitamin B12 deficiency. ${ }^{20}$ It is unclear whether the mechanism is viral or metabolic. $^{21}$

At the very least it may be asked whether the associated vitamin B12 deficiency from any cause is aggravating the underlying MS or hindering the process of remyelination? For many years vitamin B12 injections have been used occasionally for placebo purposes in the treatment of MS. Although some patients are impressed with their apparent neurological benefit, there is no controlled support for this conviction. ${ }^{22}$ Clearly, controlled treatment studies will be required in patients with $M S$ who have been separated into those with or without a disturbance of vitamin B12 metabolism.

These predominantly clinical observations should stimulate further basic research on the role of vitamin B12 and methylation in myelin formation and integrity and in demyelinating disorders. Little is known about the requirements of the central nervous system for vitamin B12, nor the forms in which it is needed or mechanisms by which it is transported across the blood-brain barrier. Such research would assist in the evaluation of the most appropriate form, dose or duration of therapy for optimum treatment of vitamin $\mathrm{B} 12$ related neurological disorders. $^{2324}$

Department of Neurology,

E H REYNOLDS

Maudsley and King's College Hospitals,

Denmark Hill, London

1 Leichtenstern O. Progressive perniciose Anamie bei Tabeskranken. Dtsch Med Wochenschr 1884;10:849-50.

2 Reynolds $\mathrm{EH}$. Folic acid, vitamin $\mathrm{Bl} 2$, and the nervous system: Historical aspects. In: Botez MI, Reynolds EH, eds. Folic acid in neurology, psychiatry, aspects. In: Botez MI, Reynolds EH, eds. Folic acid in neurolog

3 Shorvon SD, Carney MWP, Chanarin I, Reynolds EH. The neuropsychiatry of megaloblastic anaemia. Brit Med f 1980;281:1036-8.

4 Reynolds EH, Linnell JC. Vitamin Bl2 deficiency, demyelination and multiple sclerosis. Lancet 1987;ii: 920 .

5 Reynolds EH, Linnell JC, Faludy JE. Multiple sclerosis associated with vitamin B12 deficiency. Arch Neurol 1991;48:808-11.

6 Chanarin I. The megaloblastic anaemias. Oxford: Blackwell, 1979.

7 Crellin RF, Bottiglieri T, Reynolds EH. Multiple sclerosis and macrocytosis. Acta Neurol Scand 1990;81:388-91.

8 Najim Al-Din AS, Khojali M, Habbosh H, Farah S, Idris AR, Al-Muhtasib F. Macrocytosis in multiple sclerosis: a study in $\mathbf{8 2}$ de novo Arab patients. f Neurol Neurosurg Psychiatry 1991;54:415-6.

9 Plum CM, Fog T. Studies in multiple sclerosis. Acta Psychiat Neurol Scand 1959;34 (suppl 128):13-8.

10 Prineas J. Red blood cell size in multiple sclerosis. Acta Neurol Scand 1968;44:81-90.

11 Reynolds EH, Bottiglieri T, Laundy M, Crellin RF, Kirker SG. Vitamin B12 metabolism in multiple sclerosis. Arch Neurol 1992 (In press)

12 Carmel R, Herbert V. Deficiency of vitamin B12-binding alpha globulin in two brothers. Blood 1969;33:1-12.

13 Sigal SH, Hall CA, Antel JP. Plasma R-binder deficiency and neurologic disease. New Engl f Med 1987;317:1330-2.

14 Njist TQ, Hommes OR, Weavers RA, Schoonder-Waldt HC, DeHaan AFJ. Vitamin B12 and folate levels of serum and cerebrospinal fluid in multiple sclerosis and other neurological disorders. $\mathcal{f}$ Neurol Neurosurg 1990;53:951-4

15 Allen RH, Stabler SP, Savage DG, Lindenbaum J. Diagnosis of cobalamin deficiency I: Usefulness of serum methylmalonic acid and total homocysteine concentrations. Am $¥$ Hematol 1990;34:90-8.

16 Bottiglieri T. $S$-adenosylmethionine, folate, methylation and monoamine metabolism in neuropsychiatry: clinical and experimental studies. PhD Thesis submitted to London University, 1992.

17 Surtees R, Leonard J, Austin S. Association of demyelination with deficiency of cerebrospinal-fluid S-adenosylmethionine in inborn errors of methyl-transfer pathway. Lancet 1991;338:1550-4.

18 Carmel R, Watkins D, Goodman SI, Roenblatt DS. Hereditary defect of cobalamin metabolism ( $c b l \mathrm{G}$ Mutation) presenting as a neurologic disorder in adulthood. New Engl $\mathcal{F}$ Med 1988;318:1738-41.

19 Ransohoff RM, Jacobsen DW, Green R. Vitamin B12 deficiency and multiple sclerosis. Lancet 1990;335:1285-6.

20 Petito CK, Navia BA, Eun-Sook C, Jordan BD, George DC, Price RW. Vacuolar myelopathy pathologically resembling subaçute combined degeneration in patients with the acquired immunodeficiency syndrome. New Engl $\mathcal{F}$ Med 1985;312:874-9.

21 Keatingf JN, Trimble KC, Mulcahy F, Scott JM, Weir DG. Evidence of brain methyltransferase inhibition and early brain involvement in HIVbrain methyltransferase inhibition and early

22 Simpson CA, Newell DJ, Miller H. The treatment of multiple sclerosis with massive doses of hydroxocobalamin. Neurology 1967;15:599-603.

23 Reynolds EH. Neurology of vitamin B12 deficiency. Lancet 1976;ii 832-3.

24 Lazar GS, Carmel R. Cobalamin binding and uptake in vitro in the human central nervous system. $\mathcal{F}$ Lab Clin Med 1981;97:123-3. 